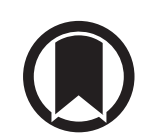

CrossMark

\title{
Detection and monitoring of lung inflammation in cystic fibrosis during respiratory tract exacerbation using diffusion-weighted magnetic resonance imaging
}

\author{
Pierluigi Ciet ${ }^{1,2,3}$, Silvia Bertolo ${ }^{3}$, Mirco Ros $^{4}$, Eleni Rosalina Andrinopoulou ${ }^{5}$, \\ Valentina Tavano ${ }^{3}$, Francesca Lucca ${ }^{6}$, Thorsten Feiweier ${ }^{7}$, Gabriel P. Krestin ${ }^{1}$, \\ Harm A.W.M. Tiddens ${ }^{1,2}$ and Giovanni Morana ${ }^{3}$
}

Affiliations: 'Dept of Radiology, Erasmus Medical Center, Rotterdam, the Netherlands. ${ }^{2}$ Dept of Pediatrics, Respiratory Medicine and Allergology, Erasmus Medical Center, Rotterdam, the Netherlands. ${ }^{3}$ Dept of Radiology, Ca'Foncello Regional Hospital, Treviso, Italy. ${ }^{4}$ Dept of Pediatrics, Ca'Foncello Regional Hospital, Treviso, Italy. ${ }^{5}$ Dept of Biostatistics, Erasmus Medical Center, Rotterdam, the Netherlands. 'Dept of Women's and Children's Health, University of Padova, Padova, Italy. ${ }^{7}$ Siemens Healthcare GmbH, Erlangen, Germany.

Correspondence: Giovanni Morana, Radiology Department, General Hospital Ca' Foncello, 31100 Treviso, Italy. E-mail: giovanni.moranadaulss2.veneto.it

@ERSpublications

DWI is a promising imaging method to detect and monitor the severity of inflammation during RTE in CF patients http://ow.ly/Ulcc30c0ebU

Cite this article as: Ciet P, Bertolo S, Ros M, et al. Detection and monitoring of lung inflammation in cystic fibrosis during respiratory tract exacerbation using diffusion-weighted magnetic resonance imaging. Eur Respir J 2017; 50: 1601437 [https://doi.org/10.1183/13993003.01437-2016].

ABSTRACT The aim was to investigate whether diffusion-weighted magnetic resonance imaging (DWI) detects and monitors inflammatory and lung function changes during respiratory tract exacerbations (RTE) treatment in patients with cystic fibrosis (CF).

29 patients with RTE underwent DWI pre- and post-antibiotic treatment. A control group of 27 stable patients, matched for age and sex, underwent DWI with the same time gap as those undergoing RTE treatment. Clinical status and lung function were assessed at each DWI time point. The CF-MRI scoring system was used to assess structural lung changes in both CF groups.

Significant reduction in the DWI score over the course of antibiotic treatment $(p<0.0001)$ was observed in patients with RTE, but not in the control group. DWI score had a strong inverse correlation with clinical status $(\mathrm{r}=-0.504, \mathrm{p}<0.0001)$ and lung function $(\mathrm{r}=-0.635, \mathrm{p}<0.0001)$ in patients with RTE. Interestingly, there were persistent significant differences in the CF-MRI score between the RTE and control group at both baseline and follow-up ( $p<0.001$ ), while the differences in DWI score were only observed at baseline $(p<0.001)$.

DWI is a promising imaging method for noninvasive detection of pulmonary inflammation during RTE, and may be used to monitor treatment efficacy of anti-inflammatory treatment.

This article has supplementary material available from erj.ersjournals.com

Received: July 192016 | Accepted after revision: April 102017

Support statement: The study was supported by the Italian Cystic Fibrosis Foundation (FFC), grant number FFC\#25/ 2011 (www.fibrosicisticaricerca.it/progetto/ffc-25-2011-dwi-un-nuovo-strumento-per-valutare-linfiammazione-nei-pazienticon-fibrosi-cistica-con-esacerbazione-respiratoria/). The sponsor had no role in the design of the study, the collection and analysis of the data, or the preparation of the manuscript. Funding information for this article has been deposited with the Crossref Funder Registry.

Conflict of interest: Disclosures can be found alongside this article at erj.ersjournals.com

Copyright @ERS 2017 


\section{Introduction}

Cystic fibrosis (CF) lung disease is characterised by chronic airways infection and inflammation, respiratory tract exacerbation (RTE) and progressive lung damage, starting in early childhood [1]. RTE is characterised by acute viral infections with more severe symptoms, reduced pulmonary function test (PFT) parameters and radiographic changes [2]. During RTE, it would be of great value to localise and monitor inflammatory changes to assess the efficacy of treatment [3]. To date, such information cannot be accurately obtained by physical examination, laboratory tests or PFT parameters because of their limited sensitivity to regional abnormalities [4].

Structural changes related to RTE are mostly assessed by chest radiography (CXR) or chest computed tomography (CT), depending on the centre [5]. CXR is the first imaging method used for RTE because of its low-cost and availability. CXR is not useful, however, in determining whether a patient with CF is having a RTE [6]. In contrast, chest CT localises and quantifies early regional structural and inflammatory lung changes [7]. However, short-term monitoring with CT for RTE is limited by radiation exposure, particularly in children [8]. To date, only a few studies are available where imaging techniques were used to monitor inflammatory changes in CF [9]. Positron emission tomography (PET)-CT has been used in a few studies, but its use is restricted by high radiation exposure and costs [10]. As an alternative to PET-CT, the lung clearance index (LCI) and fraction of exhaled nitric oxide (FENO) have been used to quantify lung inflammation, but, as with spirometry, they do not provide regional information [11, 12]. Hence, a reliable and radiation-free technique to localise and monitor lung inflammatory changes during RTE is still needed.

Magnetic resonance imaging (MRI) [13] can fulfil the requirements of a reliable and safe monitoring tool for RTE. Since MRI does not employ ionising radiation, it allows safe short-term monitoring, as needed for RTE follow-up [13]. Among the available MRI techniques, diffusion-weighted MRI (DWI) is accurate in the identification of inflammation in various organs [14] and can provide functional information regarding inflammation [14]. To date, DWI has been mostly used to characterise malignant thoracic lesions [15]. Recently, DWI has been used to discriminate between lung cancer and post-obstructive pneumonia [16], and to detect lung alterations in patients with primary antibody deficiencies [17].

For these reasons, we recently conducted a cross-sectional study in a group of stable patients with $\mathrm{CF}$, where DWI showed foci of high signal ("hotspots"), which only in part overlapped the structural lung changes on morphological CT or MRI [18]. We also observed that DWI scores had a strong correlation with radiological and clinical parameters indicators of CF lung disease severity [18].

Driven by these compelling results, we sought to investigate whether DWI: 1) is able to differentiate between stable patients with CF and those with RTE; 2) is able to track changes over the course of RTE treatment; 3) correlates with established clinical markers of CF lung disease severity and 4) shows a relationship with structural lung changes established by CF MRI score during RTE.

\section{Material and methods}

Study design and patient enrolment

This single-centre prospective study was approved by the local ethics committee (protocol no. 314/ AULSS9). Following informed consent, patients with CF admitted for RTE treatment underwent a study protocol that included clinical examination, spirometry and MRI on the same day. For each RTE patient enrolled, the next suitable CF subject without RTE in the previous 6 months was selected for the control group. Controls were CF subjects recruited during the annual follow-up and matched with RTE subjects for age, sex and mean forced expiratory volume in $1 \mathrm{~s}$ (FEV1) in the previous 6 months before the visit. General inclusion and exclusion criteria for cases and controls are described in table 1.

Patients with RTE repeated the baseline study protocol upon completion of intravenous antibiotic treatment. Similarly, control subjects repeated the study protocol over the same median time interval as the RTE group.

\section{Clinical examination, laboratory and pulmonary function tests}

Clinical examination included physical examination and weight and height measurements. Spirometry was performed according to "ATS/ERS guidelines" using a Masterscope (Jaeger-Carefusion, Hoechberg, Germany) spirometer [19]. The following parameters were recorded: forced vital capacity (FVC), FEV1 \% predicted and forced expiratory flow at $25-75 \%$ of FVC (FEF25-75).

RTE was defined and scored according to the Rosenfeld criteria for pulmonary exacerbation (PEX score) [20]. The criteria and the scoring system are listed in supplementary table S1. 


\section{MRI protocol}

Each subject in the RTE group underwent a chest MRI at admission (baseline) and at the end of antibiotic treatment (follow-up). The same median time interval ( $\sim 20$ days) was used for subjects in the control group.

MRI was performed at 1.5 tesla (MAGNETOM Avanto, Siemens Healthcare, Erlangen, Germany) using morphological and functional sequences. Morphological sequences included axial and coronal end-expiratory triggered PROPELLER (BLADE@SIEMENS) with proton density (PD) weighting [21], and breath-hold axial and coronal SSFP (TrueFISP@SIEMENS).

The DWI data were acquired in the axial plane using a DWI single-shot echo-planar imaging (EPI) sequence prototype. Detailed description of the DWI protocol is provided in the supplementary material.

\section{Image analysis}

MRI images were anonymised and evaluated in random order by two radiologists ( $\mathrm{V}$. Tavano and S. Bertolo), with 4 and 8 years of experience in thoracic MRI scoring respectively. Both radiologists were blinded to any clinical information and to each other's MRI assessment. Morphological magnetic resonance images were scored with the CF MRI scoring system. Diffusion-weighted images were scored in random order using a semi-quantitative scoring approach that had been used in previous studies [22, 23]. DW images were also assessed quantitatively with apparent diffusion coefficient (ADC) maps [24]. A more detailed description of the CF and DWI scoring systems is provided in the supplementary table S2.

\section{Statistical analysis}

Descriptive statistics, Q-Q plots and the Shapiro-Wilk test were used to test data normality. To assess baseline difference between the groups, we used the unrelated t-test and the Mann-Whitney U-test. To assess difference between baseline and follow-up MRI, we performed the related t-test and Wilcoxon test.

Inter- and intra-observer agreement for the CF-MRI and DWI scoring methods was assessed using the intraclass correlation coefficient (ICC) and Bland-Altman plots.

Pearson's correlation was used to measure the association between DWI score and clinical, spirometry and radiological parameters reflecting disease severity. These parameters included PEX score, FEV1 \% pred, FVC, FEF25-75 and total CF-MRI score (CFMRI $\left.\mathrm{TOT}_{\mathrm{TO}}\right)$. Multiple comparisons were adjusted using the Bonferroni correction. According to Cohen's criteria, correlations with $r$ between 0.10 and 0.29 are considered weak, between 0.30 and 0.49 moderate and above 0.50 strong [25].

Receiver operating characteristic (ROC) curves were used to determine accuracy of DWI to detect patient with RTE at baseline. Accuracy was expressed as area under the curve (AUC).

Finally, using mixed-effects models the outcome total DWI score was tested for the following covariates: time (baseline versus follow-up)+group (RTE versus control)+time ${ }^{\star}$ group, observer (scores observer 1 versus scores observer 2 ) and observer ${ }^{\star}$ time. The covariates "time ${ }^{\star}$ group" and "observer ${ }^{\star}$ time" specify the factorial interaction between time points within the group and observers. Differences were considered statistically significant if p-value was $<0.05$. Statistical analysis was performed with SPSS (version 20.0, SPSS, Chicago, IL, USA) and R (version 3.1.3, the R foundation for statistical computing, Vienna, Austria).

\section{Results}

Patients' characteristics

56 patients with CF were enrolled in the study (mean age 24 years, range 12-58 years, 35 female). 29 subjects were included in the RTE group (mean age 24 years, range 13-48 years, 20 female) and 27

\section{TABLE 1 General inclusion and exclusion criteria for both groups}

\section{Inclusion criteria}

Proven CF as evidenced by positive sweat test or gene mutation Ability to perform reproducible manoeuvres with spirometry Ability to comply with MRI procedures FEV $1 \geqslant 40 \%$ pred

Age $\geqslant 8$ years

\section{Exclusion criteria}

Chronic oxygen therapy Isolation of Burkholderia cepacia Lung transplantation Participation in another trial Any contraindications to MRI Pregnancy

Not able or not willing to give consent Allergic bronchopulmonary aspergillosis

CF: cystic fibrosis; MRI: magnetic resonance imaging; FEV1: forced expiratory volume in $1 \mathrm{~s}$. 
subjects in the control group (mean age 23 years, range 12-58, 15 female). The median (1st and 3rd quartile) time gap between baseline and follow-up MRI was 21 (16-35) and 15 (14-21) days for RTE patients and controls respectively. Subjects were correctly matched for age and sex between the groups. PEX score $(p<0.001)$, DWI score $(p=0.002)$ and ADC $(p<0.001)$ were significantly different between the RTE and control group at baseline, but not at follow-up. CF-MRI scores were significantly higher at both time points for the RTE relative to the control group. The patients' characteristics, spirometry, CF-MRI, DWI scores and ADC are summarised in table 2.

\section{Differences between baseline and follow-up MRI}

The RTE group showed significant differences between baseline and follow-up MRI for spirometry, PEX score, CF-MRI score, DWI score and ADC (all $\mathrm{p}<0.0001$ ). Figures 1 and 2 show DWI changes between baseline and follow-up in patients of the RTE group. In contrast, the control group did not show significant differences between baseline and follow-up for all parameters. Table 3 summarises differences between baseline and follow-up MRI within the groups. Supplementary figure S1 shows changes of PEX score, FEV1 and DWI score between the groups in time.

\section{Correlations}

The DWI score had a strong inverse correlation with FEV1 and FEF25-75 at baseline (FEV1 $\mathrm{r}=-0.635$; FEF25-75 $\mathrm{r}=-0.611$; all $\mathrm{p}<0.0001$ ) and follow-up (FEV1 $\mathrm{r}=-0.633$; FEF25-75 $\mathrm{r}=-0.626$; all $\mathrm{p}<0.0001$ ) (table 3). DWI score had a strong positive correlation with PEX score at baseline $(\mathrm{r}=0.504, \mathrm{p}<0.0001)$, but not at follow-up ( $\mathrm{r}=0.364, \mathrm{p}=0.005$; not significant after Bonferroni) (table 3 ). ADC had a strong negative correlation with PEX score at baseline $(\mathrm{r}=-0.634, \mathrm{p}<0.0001)$, but not at follow-up $(\mathrm{r}=-0.43, \mathrm{p}=0.766)$ (table 3). DWI score had a strong positive correlation with $\mathrm{CFMRI}_{\mathrm{TOT}}$ at baseline $(\mathrm{r}=0.809, \mathrm{p}<0.0001)$ and follow-up ( $\mathrm{r}=0.767, \mathrm{p}<0.0001)$ (table 3).

\section{ROC curves}

DWI score had a fair diagnostic accuracy (AUC 0.73, 95\% CI 0.59-0.86, p=0.003). For the DWI score a cut-off of 14 had a sensitivity of $73 \%$ and a specificity of $68 \%$. ADC maps had a good diagnostic accuracy (AUC $0.818,95 \%$ CI $0.69-0.94, \mathrm{p}<0.0001$ ). For the ADC maps, a cut-off value of $1.45 \times 10^{-3} \mathrm{~mm}^{2} \cdot \mathrm{s}^{-1}$ had a sensitivity of $72 \%$ and a specificity of $84 \%$. Supplementary figure S2 shows ROC curves for DWI score and ADC to identify RTE patients using the PEX score as reference.

TABLE 2 Patient characteristics, pulmonary function tests, cystic fibrosis magnetic resonance imaging score (CF-MRI), diffusion-weighted magnetic resonance imaging (DWI) scores and apparent diffusion coefficient (ADC)

\begin{tabular}{|c|c|c|c|}
\hline Parameters & Controls & RTE & p-value \\
\hline Age years & $18.50(15-31)$ & $21(17-36)$ & 0.3 \\
\hline Males/females $n / n$ & $12 / 15$ & $9 / 20$ & 0.2 \\
\hline FEV 16 months before $t_{0}$ & $85.9 \pm 25.4$ & $69.7 \pm 21.7$ & $0.011 *$ \\
\hline FVC 6 months before $t_{0}$ & $97.3 \pm 14.2$ & $85.7 \pm 14.9$ & $0.004^{*}$ \\
\hline FEV 1 at $t_{0}$ & $88.2 \pm 17.6$ & $70.7 \pm 19.5$ & $0.001 *$ \\
\hline$F V C$ at $t_{0}$ & $98.6 \pm 13.3$ & $81.9 \pm 21.8$ & $0.01 *$ \\
\hline FEF $25-75$ at $t_{0}$ & $68.8 \pm 31.8$ & $44.2 \pm 23.8$ & $0.002^{*}$ \\
\hline FEV 1 at $t_{1}$ & $88.3 \pm 18.9$ & $77.3 \pm 19.5$ & $0.036^{*}$ \\
\hline FVC at $t_{1}$ & $96.7 \pm 14.2$ & $86.9 \pm 15.6$ & $0.016^{*}$ \\
\hline$F_{E F} 25-75$ at $t_{1}$ & $68.6 \pm 33.4$ & $52.6 \pm 27.9$ & 0.053 \\
\hline PEX at $t_{0}$ & 0 & $4.5(4.1-5.9)$ & $<0.001 *$ \\
\hline PEX at $t_{1}$ & 0 & $1.2(0-1.2)$ & 0.1 \\
\hline CF-MRI at $t_{0}$ & $16.2(11-36)$ & $42(25-61)$ & $<0.001 *$ \\
\hline CF-MRI at $t_{1}$ & $16(1-34.5)$ & $39(18-56)$ & $0.002^{*}$ \\
\hline DWI at $t_{0}$ & $7(0-16.7)$ & $22(9-26)$ & $0.002^{*}$ \\
\hline DWI at $t_{1}$ & $9(0-17.7)$ & $13(5-19)$ & 0.179 \\
\hline ADC at $t_{0} \times 10^{-3} \mathrm{~mm}^{2} \cdot \mathrm{s}^{-1}$ & $1.55(1.49-1.64)$ & $1.26(1.21-1.46)$ & $<0.001 *$ \\
\hline ADC at $t_{1} \times 10^{-3} \mathrm{~mm}^{2} \cdot \mathrm{s}^{-1}$ & $1.56(1.48-1.61)$ & $1.57(1.35-1.63)$ & 0.9 \\
\hline
\end{tabular}

Data are presented as median (interquartile range) or mean \pm SD. FEV1: forced expiratory volume in $1 \mathrm{~s}$; FVC: functional vital capacity; FEF25-75: forced expiratory flow at 25-75\% of FVC; PEX: pulmonary exacerbation score. *: significant differences between the groups. 

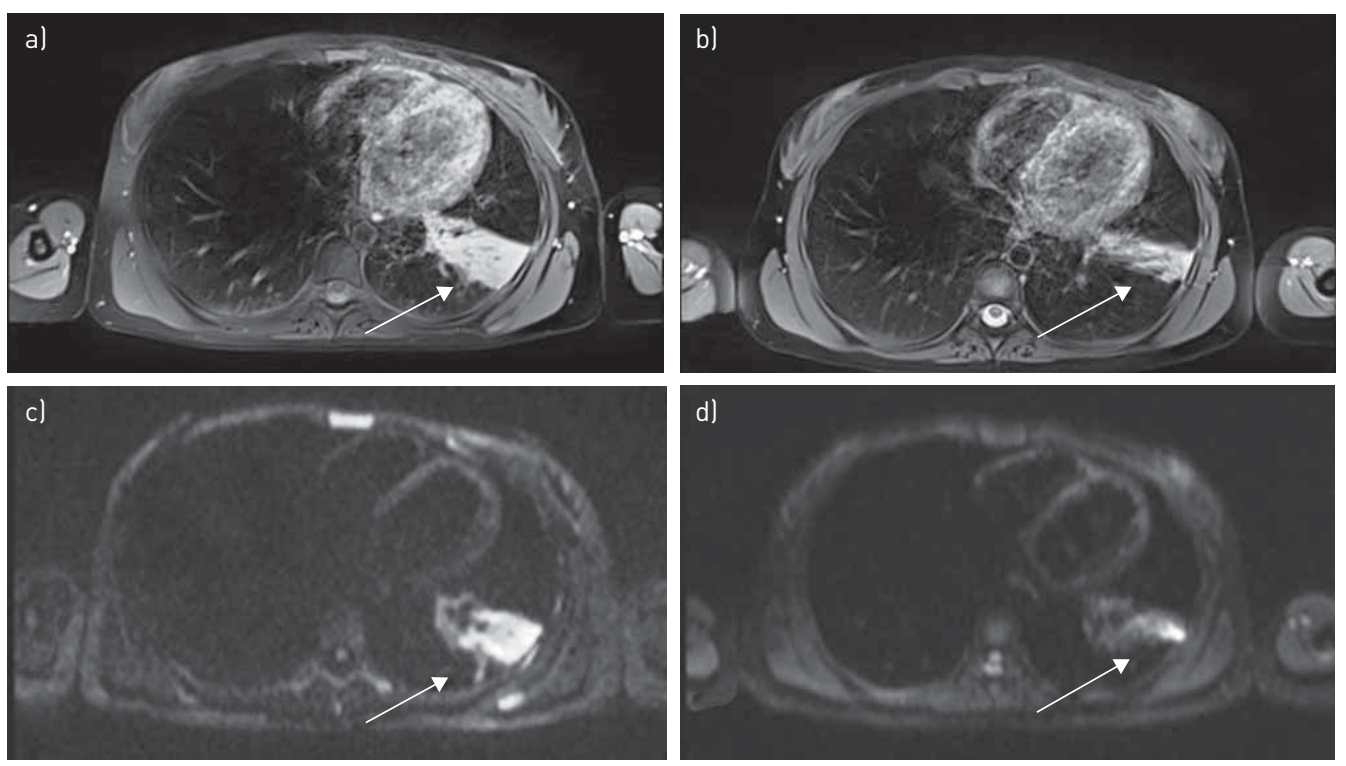

FIGURE 1 Diffusion-weighted magnetic resonance imaging (DWI) changes pre-(a, c) and post- (b, d) respiratory tract exacerbation (RTE) treatment in cystic fibrosis patient with RTE. Left column is imaging at baseline and right column is at follow-up. The upper row displays morphological free-breathing BLADE (Siemens) images, while the lower row is diffusion-weighted images at $b=800 \mathrm{~mm} \cdot \mathrm{s}^{-2}$. Note that left lower consolidation larrow in a) with high DWI signal (arrow in c) became smaller after RTE treatment (arrow in b) and DWI signal also reduced larrow in d). Total DWI score at baseline and follow-up was 7 and 4, respectively. ADC value at baseline and follow-up was 1.25 (sD=0.13) and 1.54 (sD=0.13).

\section{Mixed-model analysis}

Mixed-model analysis showed that total DWI score outcome was significantly different between the groups (group, value 13.3 DWI score points, SE 3.1, p=0.0003) and that it significantly reduced between baseline and follow-up MRI for the RTE group (group ${ }^{\star}$ time, value -5.2 DWI score points, SE $1.4, \mathrm{p}=0.0003$ ). There
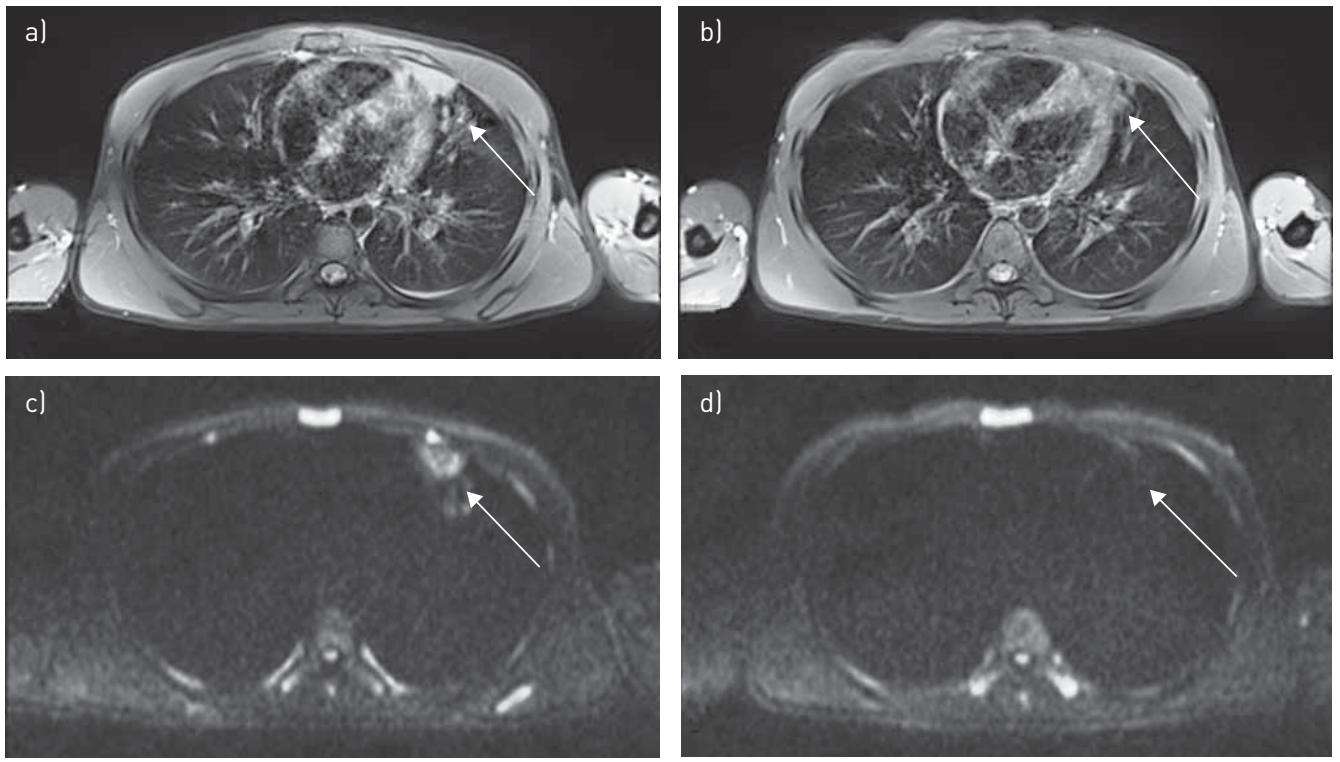

FIGURE 2 Relation between morphological and functional lung changes during respiratory tract exacerbations in a cystic fibrosis patient. Left column is imaging at baseline and right column is at follow-up. The upper row contains morphological free-breathing BLADE (Siemens) images (a, b), while the lower row is diffusion-weighted images at $b=800 \mathrm{~mm} \cdot \mathrm{s}^{-2}(\mathrm{a}, \mathrm{c})$. Note small consolidation in the lingula (arrow in a) with high diffusion-weighted magnetic resonance imaging (DWI) signal (arrow in c). After respiratory tract exacerbation treatment this consolidation is still present (arrow in b), but the DWI signal has disappeared (arrow in d), showing a discordant pattern between morphological and functional images. Total DWI score at baseline and follow-up was 19 and 12, respectively. ADC value at baseline and follow-up was 1.28 (SD=0.12) and $1.47(\mathrm{SD}=0.16)$. 
TABLE 3 Differences in spirometry, clinical and magnetic resonance imaging (MRI) parameters between baseline and follow-up

\section{Parameters}

Controls

\begin{tabular}{lc}
\cline { 2 - 2 } & Baseline \\
\hline FEV 1 & $88.2 \pm 17.6$ \\
FVC & $98.6 \pm 13.3$ \\
FEF25-75 & $68.8 \pm 31.8$ \\
PEX & 0 \\
CF-MRI & $16.2(11-36)$ \\
DWI score & $7(0-16.7)$ \\
ADC $\times \mathbf{1 0}^{-3} \mathbf{m m}^{2} \cdot \mathbf{s}^{-1}$ & $1.55(1.49-1.64)$ \\
ROI n & $1.81 \pm 1.6$ \\
ROI area $\mathbf{m m}^{2}$ & $55.36 \pm 37.37)$
\end{tabular}

$\begin{array}{cc}\text { Follow-up } & \text { p-value } \\ 88.3 \pm 18.9 & 0.976 \\ 96.7 \pm 14.2 & 0.33 \\ 68.6 \pm 33.4 & 0.948 \\ 0 & \mathrm{~ns} \\ 16(1-34.5) & 0.824 \\ 9(0-17.7) & 0.652 \\ 1.56(1.48-1.61) & 0.178 \\ 1.68 \pm 1.49 & \mathrm{~ns} \\ 58.26 \pm 39 & \mathrm{~ns}\end{array}$

RTE

\begin{tabular}{ccc}
\multicolumn{3}{c}{ RTE } \\
\hline Baseline & Follow-up & p-value \\
\hline $70.7 \pm 19.5$ & $77.3 \pm 19.5$ & $<0.0001^{*}$ \\
$81.9 \pm 21.8$ & $86.9 \pm 15.6$ & $<0.0001^{*}$ \\
$44.2 \pm 23.8$ & $52.6 \pm 27.9$ & $<0.0001^{*}$ \\
$4.5(4.1-5.9)$ & $1.2(0-1.2)$ & $<0.0001^{*}$ \\
$42(25-61)$ & $39(18-56)$ & $<0.0001^{*}$ \\
$22(9-26)$ & $13(5-19)$ & $<0.0001^{*}$ \\
$1.26(1.21-1.46)$ & $1.57(1.35-1.63)$ & $<0.0001^{*}$ \\
$3.09 \pm 1.5$ & $2.59 \pm 1.7$ & $<0.005^{*}$ \\
$132.7(90.6-232)$ & $76.9(50.2-162.7)$ & $<0.0001^{*}$
\end{tabular}

Data are presented as median (interquartile range) or mean \pm SD. FEV1: forced expiratory volume in $1 \mathrm{~s}$; FVC: functional vital capacity; FEF25-75: forced expiratory flow at $25-75 \%$ of FVC; PEX:pulmonary exacerbation score; CF-MRI:cystic fibrosis magnetic resonance imaging score; DWI score: diffusion-weighted magnetic resonance imaging total score; ADC:apparent diffusion coefficient expressed as $10^{-3} \mathrm{~mm}^{2} \cdot \mathrm{s}^{-1}$; $\mathrm{ROI}: \mathrm{region}$ of interest. *: significant differences.

were no significant differences in DWI total score between the observers (observer, $\mathrm{p}=0.58$ ). Moreover, the trend of DWI total score between baseline and follow-up was the same for both observers (observer ${ }^{\star}$ time, $\mathrm{p}=0.74$ ). Figure 3 shows effect plots for total DWI score outcome based on the mixed-effects model analysis.

\section{Reliability of DWI score}

Inter-observer agreement at baseline and follow-up MRI was excellent for all sub- and total DWI scores with the ICC ranging between 0.873 and 0.918 . Also, the intra-observer agreement was excellent with the
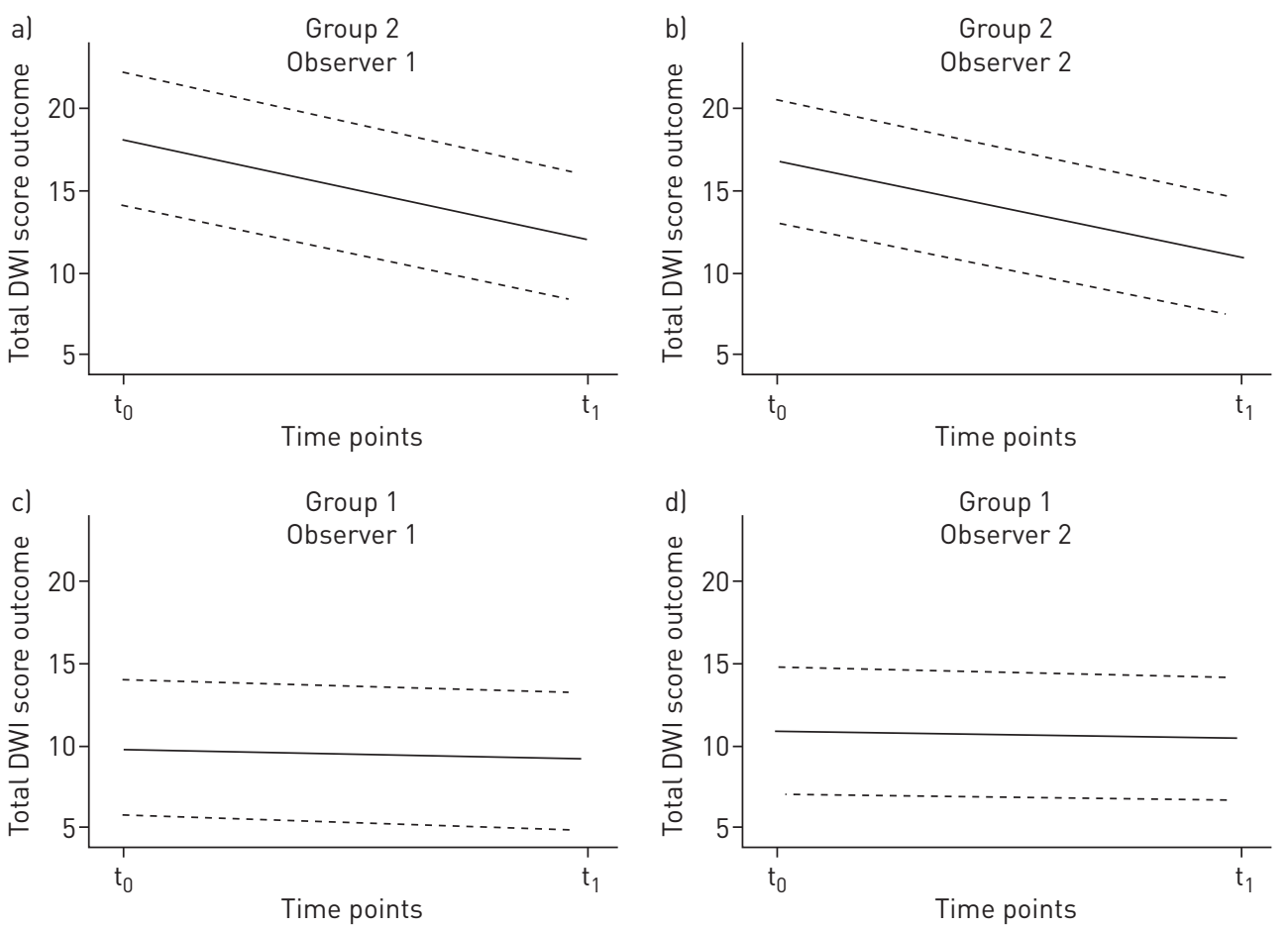

FIGURE 3 Effects plots for total diffusion-weighted magnetic resonance imaging (DWI) score outcome (total DWI) and "time" covariate. Each column represents a different observer (observer 1 versus observer 2), while each row represents control (group 1) and RTE (group 2) groups. The y-axis represents the total DWI score while the $x$-axis the baseline $\left(t_{0}\right)$ and follow-up $\left(t_{1}\right)$ time points. Each plot simulates changes in total DWI score between the time points and the groups. Dotted lines are confidence interval lines. 
ICC ranging between 0.894 and 0.947 . Larger variability for both observers was noted at follow-up MRI as shown by lower ICC values than baseline. ICC values are presented in supplementary table S3. Bland-Altman plots did not show systematic differences between the observers. Bland-Altman plots for inter- and intra-observer agreements are presented in supplementary figures 3 and 4 .

\section{Discussion}

We showed that DWI score and ADC could monitor the clinical changes related to RTE as determined by the PEX score and spirometry parameters. Our findings support the potential of DWI as a radiation-free imaging biomarker for lung inflammation.

The most interesting observation is that in patients with RTE, after intravenous antibiotic treatment, native DWI signal at high $\mathrm{b}$ values at follow-up showed a significant reduction compared with baseline (figure 1). Conversely, in the control group without RTE, no significant differences in DWI signal between follow-up and baseline were observed. This was confirmed both by DWI score and by ADC values. More importantly, these findings were also confirmed by mixed-model analysis after adjustment of confounders (i.e. observer's score), which showed a different trend of DWI score between baseline and follow-up in the two groups. These observations strongly support the concept that DWI can track inflammatory changes over the course of RTE treatment.

The second interesting finding is the relationship between CF-MRI and DWI scores. In the RTE group, the CF-MRI score was significantly lower at the end of treatment than baseline. The CF-MRI scores between the RTE and control groups were significantly higher at baseline and follow-up. This confirms the findings of prior studies that CF patients with more severe lung disease are at greater risk of developing an RTE $[26,27]$. Interestingly, the DWI score and ADC were significantly different between controls and RTE patients at baseline, but not at follow-up. This difference in DWI can be important to assess the acute changes of RTE. Prior studies have shown poor correlation between inflammatory biomarkers and reversible structural components assessed by CXR and CT [6, 28]. For example, we noticed that a consolidation or mucus plug could persist following antibiotic treatment, while the severity of local inflammation, as expressed by DWI signal, was reduced (figure 2). The ability of DWI to track inflammatory changes related to RTE could be of value to assess the treatment efficacy of RTE and to develop new treatment strategies. The sensitivity of the DWI technique may allow such studies to proceed using a relatively small number of patients.

The third important finding is the strong positive correlation of DWI score and ADC with PEX score and strong negative correlation with FEV1 and FEF25-75. It is noteworthy that, as expected, the DWI score and ADC did not correlate with PEX and CF-MRI score at the follow-up scan at the end of treatment. Additionally, the CF-MRI score had a stronger correlation with FEV1 than with the DWI score. These correlations confirm the discrepancy between structural and functional MRI lung changes during RTE, where CF-MRI better reflects the severity of structural lung disease, while DWI better depicts the inflammatory changes related to RTE.

The fourth important finding was the fair to good accuracy of DWI score and ADC to differentiate patients with CF treated for a RTE. The better diagnostic performance of ADC to define RTE patients confirms the superiority of the quantitative assessment provided by ADC map compared with the semi-quantitative DWI score.

The last noteworthy finding is that both the DWI score and ADC parameters could be scored reliably with low intra- and inter-observer variability. The strength of our DWI score was also confirmed after adjustment of confounders with mixed-model analysis.

We acknowledge some limitations to this study. Firstly, we did not obtain local samples of the DWI hotspots, which we deemed to be inflammatory. This would have required bronchoscopy, an invasive procedure not routinely performed during RTE in our institution and which is not standard clinical care in CF centres. However, the differences between the observations in the RTE and control group showed that the DWI signal was dissociated from the structural changes as quantified by the CF-MRI score. We indeed proved that only the RTE group had significant difference in the DWI score and ADC between the baseline and follow-up. Based on our promising results, we intend to perform further validation studies that include a bronchoalveolar lavage (BAL) to investigate difference between lung areas with and without hotspots and/or before and after RTE therapy. Another option would be to perform a comparison of the DWI technique with PET using PET-MRI. The radiation burden of PET-MRI is about $70 \%$ lower than PET-CT and will allow a simultaneous comparison between DWI and PET hotspots [29].

Secondly, all DWI data were acquired with respiratory gating (navigator) and interleaved mode acquisition. If motion corrections or registration techniques are not utilised, the respiratory motion might possibly 
alter ADC results and hinder DWI score evaluation. However, it has been recently shown that reliable ADC measurements can be obtained in free breathing, breath-hold and respiratory triggered DWI of lung cancer [30]. Moreover at the time of the study, we did not have the dedicated software for imaging registration that we are currently developing. In future, this software would enable a more sophisticated quantitative analysis, such as intravoxel incoherent motion [31].

Lastly, we acknowledge that this is a single-centre study; therefore, for further clinical translation, our method needs to be tested in a larger cohort multicentre study. Despite these limitations, this study represents the next important phase towards validating DWI as tool to monitor pulmonary inflammation.

\section{Conclusions}

Our study demonstrated that the DWI score and ADC changes associated with lung inflammation improved over the course of RTE treatment. Moreover, the DWI score and ADC were strongly correlated with symptom score and spirometry measures in RTE patients. The DWI score and ADC could diagnose RTE in CF patients with fair to good accuracy.

DWI is a noninvasive promising imaging method for a radiation-free detection of pulmonary inflammation during RTE in the CF population. DWI has potential to monitor the effectiveness of current and novel RTE treatment strategies to reduce lung inflammation in CF patients.

\section{Acknowledgements}

The authors thank Elizabeth Salamon, department of Respiratory Medicine, Royal Perth Hospital, for offering invaluable detailed advice on grammar and organisation of the manuscript. The researchers also wish to express their deepest gratitude to all CF patients who participated in the study.

P. Ciet, H.A.W.M. Tiddens and G. Morana participated in study concept and design. S. Bertolo, M. Ros, V. Tavano, F. Lucca, T. Feiweier and G. Morana participated in data acquisition. P. Ciet, S. Bertolo, V. Tavano, H.A.W.M. Tiddens and G. Morana undertook data analysis, interpretation and manuscript preparation and drafting. P. Ciet and E.R. Andrinopoulou undertook statistical methods and statistical data analysis. P. Ciet, T. Feiweier, G.P. Krestin, H.A.W.M. Tiddens and G. Morana undertook manuscript critique and review. All authors approved the manuscript submitted. G. Morana and P. Ciet had full access to all of the data in the study and take responsibility for the integrity of the data and the accuracy of the data analysis.

\section{References}

1 Tiddens HAWM, Donaldson SH, Rosenfeld M, et al. Cystic fibrosis lung disease starts in the small airways: can we treat it more effectively? Pediatr Pulmonol 2010; 45: 107-117.

2 Tiddens HAWM, Stick SM, Wild JM, et al. Respiratory tract exacerbations revisited: Ventilation, inflammation, perfusion, and structure (VIPS) monitoring to redefine treatment. Pediatr Pulmonol 2015; 50: Suppl. 40, S57-S65. Bhatt JM. Treatment of pulmonary exacerbations in cystic fibrosis. Eur Respir Rev 2013; 22: 205-216.

4 de Jong PA, Lindblad A, Rubin L, et al. Progression of lung disease on computed tomography and pulmonary function tests in children and adults with cystic fibrosis. Thorax 2006; 61: 80-85.

5 Eichinger M, Heussel C-P, Kauczor H-U, et al. Computed tomography and magnetic resonance imaging in cystic fibrosis lung disease. J Magn Reson Imaging 2010; 32: 1370-1378.

6 Greene KE, Takasugi JE, Godwin JD, et al. Radiographic changes in acute exacerbations of cystic fibrosis in adults: a pilot study. AJR Am J Roentgenol 1994; 163: 557-562.

7 Tiddens HAWM, Stick SM, Davis S. Multi-modality monitoring of cystic fibrosis lung disease: the role of chest computed tomography. Paediatr Respir Rev 2014; 15: 92-97.

8 Kuo W, Ciet P, Tiddens HaWM, et al. Monitoring cystic fibrosis lung disease by computed tomography. Radiation risk in perspective. Am J Respir Crit Care Med 2014; 189: 1328-1336.

9 Cohen-Cymberknoh M, Kerem E, Ferkol T, et al. Airway inflammation in cystic fibrosis: molecular mechanisms and clinical implications. Thorax 2013; 68: 1157-1162.

10 Amin R, Charron M, Grinblat L, et al. Cystic fibrosis: detecting changes in airway inflammation with FDG PET/ CT. Radiology 2012; 264: 868-875.

11 Belessis Y, Dixon B, Hawkins G, et al. Early cystic fibrosis lung disease detected by bronchoalveolar lavage and lung clearance index. Am J Respir Crit Care Med 2012; 185: 862-873.

12 Keen C, Gustafsson P, Lindblad A, et al. Low levels of exhaled nitric oxide are associated with impaired lung function in cystic fibrosis. Pediatr Pulmonol 2010; 45: 241-248.

13 Wielpütz MO, Puderbach M, Kopp-Schneider A, et al. Magnetic resonance imaging detects changes in structure and perfusion, and response to therapy in early cystic fibrosis lung disease. Am J Respir Crit Care Med 2014; 189: 956-965.

14 Attariwala R, Picker W. Whole body MRI: improved lesion detection and characterization with diffusion weighted techniques. J Magn Reson Imaging 2013; 38: 253-268.

15 Luna A, Sánchez-Gonzalez J, Caro P. Diffusion-weighted imaging of the chest. Magn Reson Imaging Clin N Am 2011; 19: 69-94

16 Deng Y, Li X, Lei Y, et al. Use of diffusion-weighted magnetic resonance imaging to distinguish between lung cancer and focal inflammatory lesions: a comparison of intravoxel incoherent motion derived parameters and apparent diffusion coefficient. Acta Radiol 2016; 57: 1310-1317.

17 Milito C, Pulvirenti F, Serra G, et al. Lung magnetic resonance imaging with diffusion weighted imaging provides regional structural as well as functional information without radiation exposure in primary antibody deficiencies. $J$ Clin Immunol 2015; 35: 491-500. 
18 Ciet P, Serra G, Andrinopoulou ER, et al. Diffusion weighted imaging in cystic fibrosis disease: beyond morphological imaging. Eur Radiol 2016; 26: 3830-3839.

19 Laszlo G. Standardisation of lung function testing: helpful guidance from the ATS/ERS Task Force. Thorax 2006; 61: 744-746.

20 Rosenfeld M, Emerson J, Williams-Warren J, et al. Defining a pulmonary exacerbation in cystic fibrosis. J Pediatr 2001; 139: 359-365.

21 Ciet P, Serra G, Bertolo S, et al. Assessment of CF lung disease using motion corrected PROPELLER MRI: a comparison with CT. Eur Radiol 2016; 26: 780-787.

22 Liu $\mathrm{H}$, Liu Y, Yu T, et al. Usefulness of diffusion-weighted MR imaging in the evaluation of pulmonary lesions. Eur Radiol 2010; 20: 807-815.

23 Uto T, Takehara Y, Nakamura Y, et al. Higher sensitivity and specificity for diffusion-weighted imaging of malignant lung lesions without apparent diffusion coefficient quantification. Radiology 2009; 252: 247-254.

24 Hagmann P, Jonasson L, Maeder P, et al. Central nervous system: state of the art understanding diffusion MR imaging techniques: from scalar imaging to diffusion. Radiographics 2006; 26: 205-224.

25 Cohen J. Statistical power analysis for the behavioral sciences. 2nd Edn. London, Routledge, 1988.

26 Loeve M, Gerbrands K, Hop WC, et al. Bronchiectasis and pulmonary exacerbations in children and young adults with cystic fibrosis. Chest 2011; 140: 178-185.

27 Sanders DB, Li Z, Brody AS. Chest computed tomography predicts the frequency of pulmonary exacerbations in children with cystic fibrosis. Ann Am Thorac Soc 2015; 12: 64-69.

28 Dakin CJ, Pereira JK, Henry RL, et al. Relationship between sputum inflammatory markers, lung function, and lung pathology on high-resolution computed tomography in children with cystic fibrosis. Pediatr Pulmonol 2002; 33: $475-482$

29 Kanal E, Barkovich AJ, Bell C, et al. ACR guidance document on MR safe practices: 2013. J Magn Reson Imaging 2013; 37: 501-530

30 Cui L, Yin JB, Hu CH, et al. Inter- and intraobserver agreement of ADC measurements of lung cancer in free breathing, breath-hold and respiratory triggered diffusion-weighted MRI. Clin Imaging 2016; 40: 892-896.

31 Le Bihan D. Intravoxel incoherent motion perfusion MR imaging: a wake-up call. Radiology 2008; 249: 748-752. 